\title{
Changes in chest X-ray findings in 1- and 2-month group after treatment initiation for suspected pulmonary tuberculosis
}

\author{
Jang Ho Lee, Ock-Hwa Kim, Yeon Joo Kim, Tae Sun Shim, and Kyung-Wook Jo
}

Division of Pulmonary and Critical Care Medicine, Department of Internal Medicine, Asan Medical Center, University of Ulsan College of Medicine, Seoul, Korea

Received: January 24, 2019

Revised : May 13, 2019

Accepted: June 12, 2019

\section{Correspondence to}

Kyung-Wook Jo, M.D.

Division of Pulmonary and Critical Care Medicine, Department of Internal Medicine, Asan Medical Center, University of Ulsan College of Medicine, 88 Olympic-ro 43-gil, Songpa-gu, Seoul 05505, Korea

Tel: $+82-2-3010-5783$

Fax: +82-2-3010-6968

E-mail: heathcliff680o@hanmail.net https://orcid.org/0000-00025949-248X
Background/Aims: Although re-evaluation of radiographic follow-up after 2 to 3 months of therapy is recommended for patients administered anti-tuberculosis medication owing to suspected pulmonary tuberculosis, reported findings are limited. Therefore, this study aimed to investigate changes in 1- and 2-month chest X-ray (CXR) findings after the treatment initiation and compared them according to the final diagnosis of tuberculosis or non-tuberculosis.

Methods: Patients who started anti-tuberculosis medication for suspected pulmonary tuberculosis were selected at a tertiary referral hospital in South Korea between January 2012 and December 2015. Changes in the 1- and 2-month CXR findings were classified as improved, unchanged, and aggravated.

Results: Among the 120 patients enrolled in the 1-month CXR group, 76 (63.3\%) had the final diagnosis of tuberculosis. Comparison between the 1-month CXR changes and diagnosis showed that the final diagnosis was tuberculosis in $81.8 \%$ (45/55), 50.0\% (26/52), and 38.5\% (5/13) of patients whose 1-month CXR was improved, unchanged, and aggravated, respectively. In the 2-month CXR group, 167 patients were enrolled, and 139 (83.2\%) of them were diagnosed with tuberculosis. Tuberculosis was the final diagnosis in $92.6 \%$ (100/108), 70.0\% (35/50), and 44.4\% (4/9) patients with improved, unchanged, and aggravated 2-month CXR findings, respectively. In patients with the final diagnosis of non-tuberculosis, nontuberculous mycobacteria and malignancy were the most common causes of improved and aggravated 1- and 2-month CXR findings, respectively.

Conclusions: Two-month CXR findings were of limited value for deciding on whether to continue anti-tuberculosis treatment. One-month CXR findings could help determine the need for further work-up.

Keywords: Antitubercular agents; Follow-up studies; Pulmonary tuberculosis; X-rays

\section{INTRODUCTION}

The World Health Organization estimated 10.0 million new cases of tuberculosis in 2018 [1]. Tuberculosis remains a major health concern in South Korea. Although tuberculosis prevalence has dramatically decreased in previous decades, its incidence is still intermediate (70 to $100 / 100,000$ people per year) during the past decade [2-4]. Appropriate diagnosis is one of the most important components to manage tuberculosis. Although culture is regarded as the gold standard in diagnosing 
tuberculosis [5], the positive rate of sputum acid-fast bacillus (AFB) culture for pulmonary tuberculosis is approximately only $70 \%$ to $80 \%$ [6-8]. Moreover, in case of negative microbial culture from respiratory samples, pulmonary tuberculosis is occasionally diagnosed based on the typical histological findings (i.e., chronic granulomatous inflammation with caseous necrosis) with good response to anti-tuberculosis medications [9]. Symptomatic patients with radiological findings compatible with active pulmonary tuberculosis have been regarded as pulmonary tuberculosis if their symptoms and radiographic lesions improved on anti-tuberculosis medication, even if they had negative AFB culture results and/or without typical histological findings. These cases have been defined as "possible" tuberculosis in previous studies and account for approximately $5 \%$ of all tuberculosis cases [10,11].

After initiating the anti-tuberculosis medication for suspected pulmonary tuberculosis, the current guidelines recommend that the attending physician should re-evaluate after a thorough clinical and radiographic follow-up study performed after 2 to 3 months of therapy [12]. This recommendation implies that clinicians should suspect diseases other than pulmonary tuberculosis if clinical or radiographic improvement is not observed. However, there is no firm evidence for this recommendation. Particularly, it is uncertain if a follow-up radiological study will be improved in all cases of tuberculosis when performed 2 months after the treatment initiation. Moreover, no study has investigated the re-evaluation based on the chest X-ray (CXR) obtained at around $<2$ months after the treatment initiation. Therefore, this study aimed to investigate changes in 1- and 2-month CXR findings after the treatment initiation and compare these changes with the final diagnosis in those who started anti-tuberculosis medication for suspected pulmonary tuberculosis.

\section{METHODS}

\section{Study subjects}

A retrospective review of the medical records at Asan Medical Center (a 2,70o-bed referral hospital in Seoul, South Korea) revealed that 2,170 patients were administered anti-tuberculosis medication for confirmed or suspected pulmonary tuberculosis between January 2012 and December 2015. Among them, 1,578 patients who had confirmed pulmonary tuberculosis with $\mathrm{My}$ cobacterium tuberculosis identified in the culture from a respiratory sample were excluded. Additionally, 85 patients were excluded based on the diagnosis in typical histological findings. Therefore, 507 patients who received $\geq 1$ dose of anti-tuberculosis medication for suspected pulmonary tuberculosis only based on radiological findings and/or clinical symptoms remained. Some patients were further excluded due to the following reasons: (1) presence of pleural effusion; (2) not treated with the standard regimen; (3) transferred to another hospital before making the final diagnosis; (4) underwent lung resection; and (5) visible lung lesion on computed tomography images but not on CXR. Finally, a total of 368 patients remained. Their medical records were retrospectively reviewed.

Changes between the baseline and 1-month or 2-month CXR findings of these patients were analyzed and then compared with their final diagnoses. The study protocol was approved by the Institutional Review Board (IRB) of the Asan Medical Center (IRB no.: 20171071), which waived the requirement for informed consent because of the retrospective nature of the analysis.

\section{One- and Two-month CXR groups}

Patients with (1) lack of CXR images taken at 1 month (between $30 \pm 7$ days); (2) therapeutic duration with anti-tuberculosis medication of < 23 days; and (3) the final diagnosis before 23 days were further excluded, and changes in the 1-month CXR finding with the final diagnosis were evaluated for the remaining patients (1-month group). Similar exclusion criteria were used on patients in the 2-month group: (1) lack of CXR images taken at 2 months (between $60 \pm 14$ days); (2) anti-tuberculosis medication treatment duration of $<46$ days; and (3) the final diagnosis was made of $<46$ days.

Among the enrolled patients, some patients did not undergo CXR at 2 months because their treatment was discontinued based on the 1-month CXR findings after the treatment initiation. Moreover, other patients underwent CXR for the first time 2 months after the treatment initiation. Therefore, the 1- and 2-month groups consisted of different patient groups. However, some patients were included in both groups if they underwent 
CXR both at 1 and 2 months.

\section{Definition of tuberculosis and non-tuberculosis}

Suspected tuberculosis was defined as pulmonary tuberculosis suspected by clinicians based on radiological and/or clinical signs, without definite evidence of tuberculosis, such as the growth of M. tuberculosis in AFB cultures, typical histological findings, or positive nucleic acid amplification test results. In patients with suspected tuberculosis, the final diagnosis was identified by the retrospective medical record review and classified into tuberculosis and non-tuberculosis. The final diagnosis of tuberculosis was made based on radiological and clinical improvement after 6 months of standard anti-tuberculosis therapy $[10,13]$. The standard therapy was defined according to guidelines as an intensive phase, consisting of isoniazid, rifampin, ethambutol, and pyrazinamide, followed by a continuation phase, consisting of isoniazid, rifampin, and ethambutol.

Non-tuberculosis was diagnosed using various diagnostic tools, such as biopsy with or without surgery, culture, and imaging follow-up. Among our study subjects, a few patients were initiated anti-tuberculosis medication based on lung biopsy results suspected with tuberculosis (such as granuloma), but their treatment was discontinued several weeks or months ( $<6$ months) after the treatment initiation because their radiologic findings have not been improved. In such cases, if (1) negative AFB culture findings were found; (2) negative results for nucleic acid amplification test performed on lung biopsy specimen were obtained; and (3) radiologic findings remained unchanged for at least 6 months, the patients were diagnosed with granuloma. In the present study, granuloma was classified into the non-tuberculosis group, because tuberculosis was defined as only when radiological and clinical improvement was noted after anti-tuberculosis therapy.

\section{Imaging evaluation}

In this study, two pulmonologists and one radiologist independently reviewed changes in CXR images without knowing the patients' final diagnosis. The baseline CXR image, which was obtained just before the initiation of anti-tuberculosis therapy, was compared with the CXR image taken at 1 or 2 months of treatment. Changes in suspected tuberculosis lesions on CXR were classified as (1) improved, (2) unchanged, and (3) aggravated. If disagreement was observed between them, a consensus decision was achieved after discussion.

\section{Data collection and statistical analysis}

The Student's $t$ test was used to compare continuous variables, and the chi-square or Fisher's exact test for categorical variables. All tests of significance were two-sided; $p$ values of $<0.05$ were considered as indicative of statistical significance. All analyses were performed using the SPSS software version 24.0 (IBM Co., Armonk, NY, USA).

\section{RESULTS}

\section{Study enrollment}

A total of 120 patients were enrolled in the 1-month CXR group after applying the exclusion criteria between January 2012, and December 2015. The exclusion criteria are presented in Fig. 1. Fig. 2 shows that 167 patients were included in the 2-month study group. A total of $47 \mathrm{pa}-$ tients underwent CXR both at 1 and 2 months after the treatment initiation and were included in both the 1and 2-month groups. Table 1 presents the baseline characteristics of 267 study subjects according to the study group.

\section{One-month CXR changes and diagnosis}

Table 2 shows the baseline characteristics of 120 patients in the 1-month group. The mean age of patients in the 1-month group was 53.2 years, and $58.3 \%$ of them were men. The final diagnosis of 120 patients was tuberculosis in 76 (63.3\%) patients and non-tuberculosis in the remaining 44 (36.7\%) patients. The baseline characteristics of the two groups were comparable, except for the differences in age and presence of malignancy (Table 2).

Compared with the baseline CXR images, 1-month images were improved changes in 55 (45.8\%), unchanged in $52(43.3 \%)$, and aggravated in 13 (10.8\%) patients. Among them, 45, 26, and five patients had a final diagnosis of tuberculosis (Fig. 1). Therefore, the final diagnosis was tuberculosis in 81.8\% (45/55), 50.0\% (26/52), and $38.5 \%$ (5/13) in patients with 1-month CXR who showed improved, unchanged, and aggravated findings, respectively. Among the 76 patients who were finally con- 


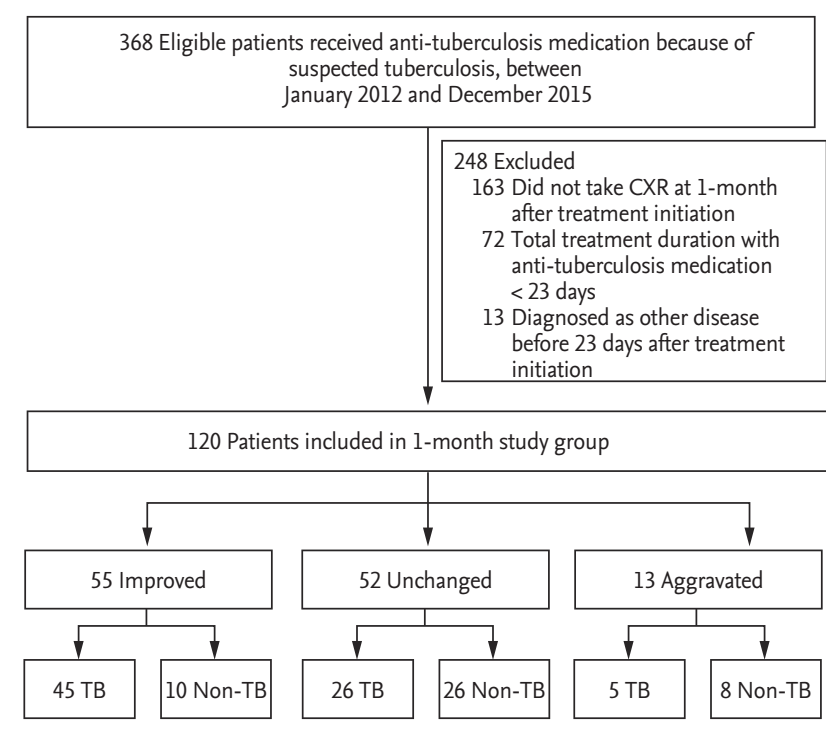

Figure 1. Study flow chart of the 1-month group. CXR, chest $\mathrm{X}$-ray; TB, tuberculosis.

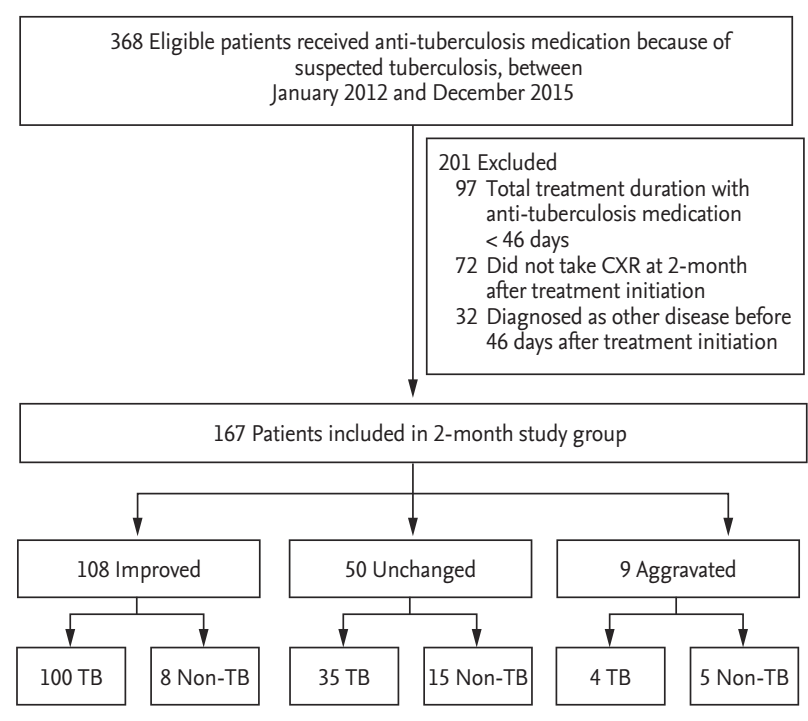

Figure 2. Study flow chart of the 2-month group. CXR, chest $\mathrm{X}$-ray; TB, tuberculosis.

Table 1. Baseline characteristics in the 267 subjects according to the study group

\begin{tabular}{|c|c|c|c|c|}
\hline Characteristic & $\begin{array}{c}\text { Total } \\
(\mathrm{n}=267)\end{array}$ & $\begin{array}{l}\text { 1-mon group } \\
(\mathrm{n}=120)\end{array}$ & $\begin{array}{c}\text { 2-mon group } \\
(n=167)\end{array}$ & $p$ value \\
\hline Age, yr & $52.1 \pm 15 \cdot 7$ & $53.2 \pm 15.2$ & $51.3 \cdot \pm 16.1$ & 0.309 \\
\hline Male sex & $167(62.5)$ & $70(58.3)$ & $97(58.1)$ & 0.966 \\
\hline Body mass index, $\mathrm{kg} / \mathrm{m}^{2}$ & $22.0 \pm 3.2$ & $21.8 \pm 3 \cdot 3$ & $22.2 \pm 3.1$ & 0.287 \\
\hline Current or past smoker & $136(50.9)$ & $55(45.8)$ & $81(48.5)$ & 0.655 \\
\hline Previous history of tuberculosis treatment & $53(19.9)$ & $24(20.0)$ & $29(17.4)$ & 0.570 \\
\hline Presence of cavity & $56(21.0)$ & $25(20.8)$ & $31(18.6)$ & 0.632 \\
\hline \multicolumn{5}{|l|}{ Underlying diseases } \\
\hline Hypertension & $60(22.5)$ & $26(21.7)$ & $34(20.4)$ & 0.788 \\
\hline Malignancy & $57(21.3)$ & $28(23 \cdot 3)$ & $29(17.4)$ & 0.211 \\
\hline Diabetes mellitus & $32(12.0)$ & $13(10.8)$ & $19(11.4)$ & 0.885 \\
\hline
\end{tabular}

Values are presented as mean $\pm \mathrm{SD}$ or number $(\%)$.

firmed with pulmonary tuberculosis, the CXR taken at 1 month showed improvement in $45(59.2 \%)$, no change in 26 (34.2\%), and aggravation in five (6.6\%) patients.

Detailed diagnoses of 44 patients in the non-tuberculosis group are described in Table 3. Nontuberculous mycobacteria (NTM) were the most common diagnoses in patients with improved or unchanged 1-month CXR findings, and malignancy was the most common cause in patients with aggravated findings. The etiologic organism was Mycobacterium avium complex (MAC) in all six patients with improved 1-month CXR finding, with the final diagnosis of NTM.

\section{Two-month CXR changes and diagnosis}

The baseline characteristics of 167 patients in the 2-month group are presented in Table 4. Among them, $139(83.2 \%)$ were finally diagnosed with tuberculosis, whereas the remaining 28 (16.8\%) were diagnosed with nontuberculous disease. No statistically significant differences in the baseline characteristics were noted, except for cavitary lesions on CXR and previous history of tuberculosis treatment (Table 4).

Compared with the baseline CXR images, the 2-month CXR image findings showed improved changes in 108 (64.7\%), no changes in 50 (29.9\%), and aggravated chang- 
Table 2. Baseline characteristics in the 1-month group according to the final diagnosis

\begin{tabular}{|c|c|c|c|c|}
\hline Characteristic & $\begin{array}{c}\text { Total } \\
(\mathrm{n}=120)\end{array}$ & $\begin{array}{c}\text { Tuberculosis } \\
(\mathrm{n}=76)\end{array}$ & $\begin{array}{c}\text { Non-tuberculosis } \\
(\mathrm{n}=44)\end{array}$ & $p$ value \\
\hline Age, yr & $53.2 \pm 15.2$ & $50.8 \pm 16.0$ & $57 \cdot 3 \cdot \pm 12.9$ & 0.018 \\
\hline Male sex & $70(58.3)$ & $42(55 \cdot 3)$ & $28(63.6)$ & 0.370 \\
\hline Body mass index, $\mathrm{kg} / \mathrm{m}^{2}$ & $21.8 \pm 3 \cdot 3$ & $22.1 \pm 3.4$ & $21.2 \pm 2.9$ & 0.130 \\
\hline Current or past smoker & $55(45.8)$ & $34(44 \cdot 7)$ & $21(47 \cdot 7)$ & 0.751 \\
\hline Previous history of tuberculosis treatment & $24(20.0)$ & $12(15.8)$ & $12(27 \cdot 3)$ & 0.130 \\
\hline Presence of cavity & $25(20.8)$ & $12(15.8)$ & $13(29 \cdot 5)$ & 0.074 \\
\hline \multicolumn{5}{|l|}{ Underlying diseases } \\
\hline Hypertension & $26(21.7)$ & $18(23.7)$ & $8(18.2)$ & 0.481 \\
\hline Malignancy & $28(23.3)$ & $12(15.8)$ & $16(36.4)$ & 0.010 \\
\hline Diabetes mellitus & $13(10.8)$ & $9(11.8)$ & $4(9.1)$ & 0.766 \\
\hline
\end{tabular}

Values are presented as mean \pm SD or number (\%).

Table 3. Detailed diagnoses of 44 patients in the 1-month group with non-tuberculosis as the final diagnosis

\begin{tabular}{|c|c|c|c|c|}
\hline Variable & Total $(n=44)$ & Improved $(n=10)$ & Unchanged $(n=26)$ & Aggravated $(\mathrm{n}=8)$ \\
\hline Nontuberculous mycobacteria & 16 & 6 & 9 & 1 \\
\hline Malignancy & 14 & o & 8 & 6 \\
\hline Granuloma $^{\mathrm{a}}$ & 4 & $\mathrm{O}$ & 4 & o \\
\hline Sarcoidosis & 2 & 1 & 1 & o \\
\hline Organizing pneumonia & 2 & 1 & 1 & ० \\
\hline Bacterial pneumonia & 1 & o & 1 & o \\
\hline Diffuse panbronchiolitis & 1 & 1 & o & o \\
\hline Castleman's disease & 1 & o & 1 & o \\
\hline Fungal pneumonia & 1 & o & o & 1 \\
\hline Necrotizing pneumonia & 1 & o & 1 & o \\
\hline Non-specific inflammation & 1 & 1 & 0 & 0 \\
\hline
\end{tabular}

${ }^{a}$ Among the patient whose biopsy result suspected with tuberculosis, the diagnosis of granuloma was made if (1) negative acid-fast bacillus culture findings were found, (2) negative results for nucleic acid amplification test performed on lung biopsy specimen were obtained, and (3) radiologic findings remained unchanged for at least 6 months.

es in nine (5.4\%) patients. Fig. 2 shows that among the patients with the 2-month CXR who showed improved, unchanged, and aggravated findings, tuberculosis was the final diagnosis in $92.6 \%$ (100/108), 70.0\% (35/50), and $44.4 \%$ (4/9), respectively. Among the 139 patients who were finally diagnosed with pulmonary tuberculosis, the 2-month CXR showed improvement in 100 (71.9\%), no change in 35 (25.2\%), and aggravation in four $(2.9 \%)$ patients.

Table 5 shows the detailed diagnoses of patients with nontuberculous disease. Among them, NTM was the most common cause of improvement in patients with the 2-month CXR findings, whereas granuloma was the most common reason for unchanged 2-month CXR findings. Among the six patients with NTM who had improved CXR findings, the etiologic organism was MAC in 83.3\% (5/6). Among the patients with aggravated changes, malignancy was the most common cause.

\section{DISCUSSION}

Current guidelines recommend that patients who have started anti-tuberculosis medication because of clinical 
Table 4. Baseline characteristics in the 2-month group according to the final diagnosis

\begin{tabular}{|c|c|c|c|c|}
\hline Characteristic & $\begin{array}{c}\text { Total } \\
(\mathrm{n}=167)\end{array}$ & $\begin{array}{l}\text { Tuberculosis } \\
\qquad(\mathrm{n}=139)\end{array}$ & $\begin{array}{l}\text { Non-tuberculosis } \\
\qquad(\mathrm{n}=28)\end{array}$ & $p$ value \\
\hline Age, yr & $51.3 \pm 16.1$ & $50.9 \pm 15.8$ & $54 \cdot 4 \pm 17.5$ & 0.326 \\
\hline Male sex & $97(58.1)$ & $79(56.8)$ & $18(64 \cdot 3)$ & 0.466 \\
\hline Body mass index, $\mathrm{kg} / \mathrm{m}^{2}$ & $22.2 \pm 3.1$ & $22.4 \pm 3.1$ & $21.2 \pm 2.8$ & 0.084 \\
\hline Current or past smoker & $81(48.5)$ & $68(48.9)$ & $13(46.4)$ & 0.810 \\
\hline Previous history of tuberculosis treatment & $29(17 \cdot 4)$ & $19(13.7)$ & $10(35 \cdot 7)$ & 0.005 \\
\hline Presence of cavity & $31(18.6)$ & $22(15.8)$ & $9(32.1)$ & 0.043 \\
\hline \multicolumn{5}{|l|}{ Underlying diseases } \\
\hline Hypertension & $34(20.4)$ & $31(22.3)$ & $3(10.7)$ & 0.205 \\
\hline Malignancy & $29(17.4)$ & $21(15.1)$ & $8(28.6)$ & 0.086 \\
\hline Diabetes mellitus & $19(11.4)$ & $18(12.9)$ & $1(3.6)$ & 0.204 \\
\hline
\end{tabular}

Values are presented as mean $\pm \mathrm{SD}$ or number (\%).

Table 5. Detailed diagnosis of 28 patients in the 2 -month group with non-tuberculosis as the final diagnosis

\begin{tabular}{|c|c|c|c|c|}
\hline Variable & Total $(\mathrm{n}=28)$ & Improved $(\mathrm{n}=8)$ & Unchanged $(\mathrm{n}=15)$ & Aggravated $(\mathrm{n}=5)$ \\
\hline Nontuberculous mycobacteria & 10 & 6 & 2 & 1 \\
\hline Malignancy & 6 & o & 4 & 3 \\
\hline Granuloma $^{\mathrm{a}}$ & 5 & o & 5 & o \\
\hline Sarcoidosis & 2 & 1 & 1 & o \\
\hline Organizing pneumonia & 1 & o & 1 & o \\
\hline Pneumoconiosis & 1 & o & 1 & o \\
\hline Castleman's disease & 1 & $\mathrm{O}$ & 1 & o \\
\hline Fungal pneumonia & 1 & o & o & 1 \\
\hline Non-specific inflammation & 1 & 1 & o & o \\
\hline
\end{tabular}

${ }^{a}$ Among the patient whose biopsy result suspected with tuberculosis, the diagnosis of granuloma was made if(1) negative acid-fast bacillus culture findings were found, (2) negative results for nucleic acid amplification test performed on lung biopsy specimen were obtained, and (3) radiologic findings remained unchanged for at least 6 months.

and/or radiological signs of tuberculosis alone should be re-evaluated after 2 to 3 months of therapy [12] because various diseases other than pulmonary tuberculosis showed similar clinical and/or radiological findings. However, no study has confirmed the appropriateness of this recommendation. To our knowledge, the present study is the first to evaluate this issue. We believe that our findings have several important clinical implications.

First, changes in the 2-month CXR findings after the treatment initiation appears to be an imperfect tool for the precise evaluation of patients with suspected tuberculosis. This is because changes noted in the 2-month
CXR findings showed inconsistent results compared with the patient's final diagnosis. Various changes in the 2-month CXR findings, including improvement, no change, and aggravation, would seem to make it difficult for an attending physician to decide whether to continue the anti-tuberculosis treatment or re-evaluate the lesion that was suspected as indicative of tuberculosis. Notably, the evidence supporting the recommendation in the current guidelines to re-evaluate at 2 to 3 months after the treatment initiation is limited [12] because it is based on one study published in 1989 [14]. That study had limitations in terms of the sample size and treatment adequacy. That is, the radiological evaluation was 
performed in 139 patients only, and anti-tuberculosis therapy consisted of isoniazid, rifampin, and ethambutol, without pyrazinamide. Moreover, rifampin was excluded in the regimen for pregnant women. Further, repeat radiographic evaluation was performed 3 months after the therapy in that study, not 2 months.

Second, our study results suggested that radiographic follow-up 1-month after the treatment initiation would be beneficial in patients suspected with pulmonary tuberculosis because 1-month CXR could be useful for judging whether re-evaluation would be necessary earlier than 2 months. We showed that among the patients who showed unchanged findings on the 1-month CXR, half of the patients' final diagnosis was non-tuberculosis. Moreover, among the patients with CXR findings that showed aggravation at 1-month, most of them had the final diagnosis of non-tuberculosis. These findings suggest that if 1-month CXR after the treatment initiation shows unchanged or aggravated findings, immediate work-up might be considered to evaluate the possibility of other diseases, such as malignancy.

Third, patients with pulmonary tuberculosis having unchanged or even aggravated findings in the 1- and/ or 2-month CXR are not especially rare. Previous studies reported that CXR uncommonly show unchanged findings compared with baseline findings even after 2 months of anti-tuberculosis treatment for pulmonary tuberculosis. For example, one report in Japan showed that $40.2 \%(29 / 72)$ of patients showed no improvement in CXR findings after 2 months of anti-tuberculous treatment [15]. How et al. [16] also showed that $35.9 \%$ of patients showed unchanged CXR findings after 8 weeks of anti-tuberculosis treatment. Moreover, some patients with pulmonary tuberculosis revealed transient aggravation on 1- or 2-month CXR even after an appropriate treatment in the present study. This is highly likely caused by temporary deterioration due to a paradoxical response, which is defined as transient radiological worsening of pre-existing tuberculosis lesions or development of new lesions not attributable to the normal course of tuberculosis following the anti-tuberculosis treatment [17]. Previous studies have shown that the incidence of paradoxical response of pulmonary tuberculosis is $2 \%$ to $10 \%$ with a median onset time of 26 to 87 days after the initiation of anti-tuberculosis treatment [18-20].
Fourth, even among those whose CXR findings were improved after treatment, the final diagnosis was not pulmonary tuberculosis in a few patients. NTMlung disease was the most common diagnosis of these patients; most of etiologic organism was MAC. The incidence of pulmonary MAC has been increasing worldwide, including in South Korea [21]. Because the symptoms and radiographic findings of MAC pulmonary disease are similar to those of pulmonary tuberculosis [22-24], pulmonary MAC lung disease should be considered as one of the differential diagnoses in patients with suspected tuberculosis when the AFB culture results are not yet available. Previously, we have shown that spontaneous negative conversion of sputum cultures occurs in approximately half of patients with MAC lung disease [25]. One of the predictors of sputum conversion is transient ( $\geq 1$ month) anti-tuberculosis medication during the initial diagnosis. Namely, the transient treatment with anti-tuberculosis medication, including rifampin and ethambutol, before the definite diagnosis of MAC lung disease may lead to improved radiological findings. The results in this study suggested that the possibility of MAC lung disease should be considered even in patients with improved radiological lesions after an anti-tuberculosis medication, given that the incidence of MAC lung disease has been increasing.

This study had several limitations. Most significantly, it was a retrospective design, was conducted at a single referral center, and included a relatively small number of cases. Second, changes in clinical symptoms were not properly assessed because the data were retrospectively collected. However, it should be noted that it was not possible to evaluate the change in clinical symptoms for most patients because most of our patients were asymptomatic at the time of tuberculosis medication initiation. Third, although no differences were observed in baseline characteristics between the 1- and 2-month groups, most of them consisted of different patients. Therefore, the diagnostic yield at 1- and 2-month CXR was not directly compared in patients with suspected tuberculosis. Fourth, a substantial proportion of patients who started anti-tuberculosis medication did not undergo the 1- or 2-month CXR follow-up examination and therefore were excluded from the analysis. Finally, the decision on whether to re-evaluate the lung lesions according to the CXR results was solely made by the attending clinicians 
without following a standard protocol.

In conclusion, CXR performed at 2 months appeared as an imperfect tool in deciding whether to continue the anti-tuberculosis medication in patients with suspected pulmonary tuberculosis who started anti-tuberculosis medication. In addition, it would be beneficial to perform CXR at 1 month after the treatment initiation so that earlier work-up can be performed to assess the possibility of diseases other than pulmonary tuberculosis in patients with unchanged or aggravated findings. Moreover, patients with the final diagnosis of tuberculosis could rarely have unchanged or even aggravated findings in the 1- and/or 2-month CXR. Finally, even in the case with improved findings at 1- or 2-month CXR, NTM lung disease may still be considered rather than pulmonary tuberculosis.

\section{KEY MESSAGE}

1. Changes in 2-month chest X-ray (CXR) findings after the treatment initiation appears to be an imperfect tool for the precise evaluation of patients with suspected tuberculosis.

2. Radiographic follow-up 1 month after the treatment initiation would be beneficial because 1-month CXR could be used to determine the need for re-evaluation.

3. Improved findings in 1- or 2-month CXR may be caused by nontuberculous mycobacteria lung disease rather than pulmonary tuberculosis.

\section{Conflict of interest}

No potential conflict of interest relevant to this article was reported.

\section{REFERENCES}

1. World Health Organization. Global tuberculosis report 2019 [Internet]. Geneva (CH): WHO, 2019 [cited 2020 Feb 3] Available from: https://www.who.int/tb/publications/ global_report/en/.

2. Kim JH, Yim JJ. Achievements in and challenges of tuberculosis control in South Korea. Emerg Infect Dis 2015;21:1913-1920.
3. Hong YP, Kim SJ, Lew WJ, Lee EK, Han YC. The seventh nationwide tuberculosis prevalence survey in Korea, 1995. Int J Tuberc Lung Dis 1998;2:27-36.

4. Shin HJ, Kwon YS. Treatment of drug susceptible pulmonary tuberculosis. Tuberc Respir Dis (Seoul) 2015;78:161167.

5. Zumla A, Raviglione M, Hafner R, von Reyn CF. Tuberculosis. N Engl J Med 2013;368:745-755.

6. Siddiqi K, Lambert ML, Walley J. Clinical diagnosis of smear-negative pulmonary tuberculosis in low-income countries: the current evidence. Lancet Infect Dis 2003;3:288-296.

7. Al-Nakeeb Z, Gupta V, Bell C, Woodhead M. Are we missing opportunities to confirm the diagnosis of tuberculosis by microbial culture? Respir Med 2013;107:2022-2028.

8. American Thoracic Society; CDC; Infectious Diseases Society of America. Treatment of tuberculosis. MMWR Recomm Rep 2003;52(RR-11):1-77.

9. Park JS, Kang YA, Kwon SY, et al. Nested PCR in lung tissue for diagnosis of pulmonary tuberculosis. Eur Respir J 2010;35:851-857.

10. Vadwai V, Boehme C, Nabeta P, Shetty A, Alland D, Rodrigues C. Xpert MTB/RIF: a new pillar in diagnosis of extrapulmonary tuberculosis? J Clin Microbiol 2011;49:2540-2545.

11. Boehme CC, Nabeta P, Hillemann D, et al. Rapid molecular detection of tuberculosis and rifampin resistance. $\mathrm{N}$ Engl J Med 2010;363:1005-1015.

12. Nahid P, Dorman SE, Alipanah N, et al. Official American Thoracic Society/Centers for Disease Control and Prevention/Infectious Diseases Society of America clinical practice guidelines: treatment of drug-susceptible tuberculosis. Clin Infect Dis 2016;63:e147-e195.

13. Kim YW, Kwak N, Seong MW, et al. Accuracy of the Xpert ${ }^{\circledR}$ MTB/RIF assay for the diagnosis of extra-pulmonary tuberculosis in South Korea. Int J Tuberc Lung Dis 2015;19:81-86.

14. Gordin FM, Slutkin G, Schecter G, Goodman PC, Hopewell PC. Presumptive diagnosis and treatment of pulmonary tuberculosis based on radiographic findings. Am Rev Respir Dis 1989;139:1090-1093.

15. Yano S, Kobayashi K, Kato K, Ikeda T. Improvement on chest X-ray findings after two months of antituberculous treatment. Kekkaku 2005;80:591-594.

16. How SH, Kuan YC, Ng TH, Razali MR, Fauzi AR. Monitoring treatment response in sputum smear positive 
pulmonary tuberculosis patients: comparison of weight gain, sputum conversion and chest radiograph. Malays J Pathol 2014;36:91-96.

17. Cheng VC, Ho PL, Lee RA, et al. Clinical spectrum of paradoxical deterioration during antituberculosis therapy in non-HIV-infected patients. Eur J Clin Microbiol Infect Dis 2002;21:803-809.

18. Cheng SL, Wang HC, Yang PC. Paradoxical response during anti-tuberculosis treatment in HIV-negative patients with pulmonary tuberculosis. Int J Tuberc Lung Dis 2007;11:1290-1295.

19. Narita M, Ashkin D, Hollender ES, Pitchenik AE. Paradoxical worsening of tuberculosis following antiretroviral therapy in patients with AIDS. Am J Respir Crit Care Med 1998;158:157-161.

20. Breen RA, Smith CJ, Bettinson H, et al. Paradoxical reactions during tuberculosis treatment in patients with and without HIV co-infection. Thorax 2004;59:704-707.

21. Ko RE, Moon SM, Ahn S, et al. Changing epidemiology of nontuberculous mycobacterial lung diseases in a tertiary referral hospital in Korea between 2001 and 2015. J Korean Med Sci 2018;33:e65.

22. Huang CT, Tsai YJ, Shu CC, et al. Clinical significance of isolation of nontuberculous mycobacteria in pulmonary tuberculosis patients. Respir Med 2009;103:1484-1491.

23. Ryu YJ, Koh WJ, Daley CL. Diagnosis and treatment of nontuberculous mycobacterial lung disease: clinicians' perspectives. Tuberc Respir Dis (Seoul) 2016;79:74-84.

24. Kwon YS, Koh WJ, Daley CL. Treatment of mycobacterium avium complex pulmonary disease. Tuberc Respir Dis (Seoul) 2019;82:15-26.

25. Hwang JA, Kim S, Jo KW, Shim TS. Natural history of Mycobacterium avium complex lung disease in untreated patients with stable course. Eur Respir J 2017;49:1600537. 